Annls limnol. 9 (1) $1973: 63-77$.

\title{
INFLUENCE DE LA TEMPERATURE ET DU pH DU MLLIEU AMBIANT \\ SUR L'ÉLECTROCARDIOGRAMME ET LES MOUVEMENTS OPERCULAIRES CHEZ LA CARPE ET LA TANCHE
}

\author{
G. DAZAROLA, R. LABAT ET J, KUGLER ${ }^{1}$.
}

\begin{abstract}
Les variations de température influencent les réactions physiologiques de la Carpe et de la Tanche plus fortement que celles dues au pH ou à l'action combinée de ces deux facteurs. La température de $25^{\circ} \mathrm{C}$ est un maximum critique au-delà duquel l'action du parasympathique se trouve modifiée. Le milieu acide inhibe l'activité cardiaque et accélère la fréquence respiratoire chez la Carpe; cette action peut être attribuée au déficit en oxygène provoqué par une baisse du $\mathrm{pH}$ sanguin.
\end{abstract}

\section{The influence of the temperature and $\mathrm{pH}$ of the surrounding environment on the electrocardiogram and opercular movements of the carp and tench.}

The physiological reactions of carp and tench are affected more strongly by temperature variations than by $\mathrm{pH}$ or the combined action of these two factors. A temperature of $25^{\circ} \mathrm{C}$ is the critical maximum above which the action of the parasympathetic nervous system is found to be modified. The acid environment inhibits cardiac activity and accelerates respiratory frequency in the carp; this action is perhaps due to an oxygen deficit caused by a fall in the level of the blood $\mathrm{pH}$.

Dans les régions tempérées, la température des eaux intérieures varie généralement entre $0^{\circ} \mathrm{C}$ et $30^{\circ} \mathrm{C}$ au cours de l'année. Les Poissons dulçaquicoles de ces régions sont bien adaptés à ces variations saisonnières de la température, à condition que ces variations soient progressives, car lorsqu'elles sont brutales, elles peuvent devenir mortelles (choc thermique). On peut done redouter que le déversement d'une grande quantité d'effluents réchauffées, surtout au moment de l'étiage, affecte directement ou indirec-

1. Université Paul-Sabatier, Laboratoire d'Écophysiologie Animale, 38, rue des Trente-Six-Ponts, 31 - Toulouse. 
tement les organismes aquatiques et déséquilibre les écosystèmes. Pour cette raison, il est important de connaître les conditions de survie des Poissons à un réchauffement artificiel du milieu ambiant, et les températures maximales compatibles avec cette survie. En outre, dans le cadre de l'écosystème, différents facteurs agissent en synergie, c'est pourquoi nous avons choisi d'étudier simultanément les variations de la température et du pH du milieu ambiant.

D'après Dussart (1966), ce sont les concentrations relatives d'anhydride carbonique, des bicarbonates, et des carbonates qui déterminent la valeur du $\mathrm{pH}$ quand l'état d'équilibre est atteint.

Dans nos régions, les ions alcalino-terreux $\left(\mathrm{Mg}^{++}\right.$et $\left.\mathrm{Ca}^{++}\right)$sont plus abondants que les ions alcalins $\left(\mathrm{Na}^{+}\right.$et $\left.\mathrm{K}^{+}\right)$, de ce fait, les carbonates et les bicarbonates sont généralement calciques. Dans les eaux riches en producteurs primaires, l'utilisation du $\mathrm{CO}_{2}$ et des bicarbonates par la photosynthèse provoque pendant le jour une élévation du $\mathrm{pH}$ (alcalinisation) tandis que pendant la nuit le rejet du $\mathrm{CO}_{2}$ respiratoire détermine le phénomène inverse (acidification). La température agissant sur la photosynthèse, les variations de pH seront plus importantes en eau chaude qu'en eau froide d'où l'intérêt d'une expérimentation dans une zone de température relativement élevée.

Labat et coll. (1969) ont montré qu'à température constante optimale, les variations de la composition ionique du milieu aquatique ont une importance prépondérante sur la physiologie des Poissons.

\section{1. - MATERIEL ET TECHNIQUES}

Nous avons utilisé pour nos expériences 106 Carpes (Cyprinus carpio L.) de trois étés dont le poids pouvait varier entre 400 et $600 \mathrm{~g}$. Parmi ces Poissons, 96 ont été utilisés pour étudier les variations de l'électrocardiogramme, 36 ont subi une vagotomie bilatérale et 20 ont servi à l'étude simultanée des modifications de I'E.C.G. et de celles de la mécanique respiratoire.

De même, nous avons expérimenté sur 54 Tanches (Tinca tinca $\mathrm{L}$.) normales et 36 Tanches vagotomisées, d'un poids moyen de 200 à $250 \mathrm{~g}$.

Tous les Poissons sont munis d'électrodes thoraciques d'après la technique de Labat et Serfaty (1963).

En ce qui concerne l'étude de la mécanique respiratoire, nous avons mis au point une technique d'enregistrement des mouvements respiratoires chez les Poissons nageant librement dans leur milieu (Dazarola, Labat et Serfaty, 1972). 

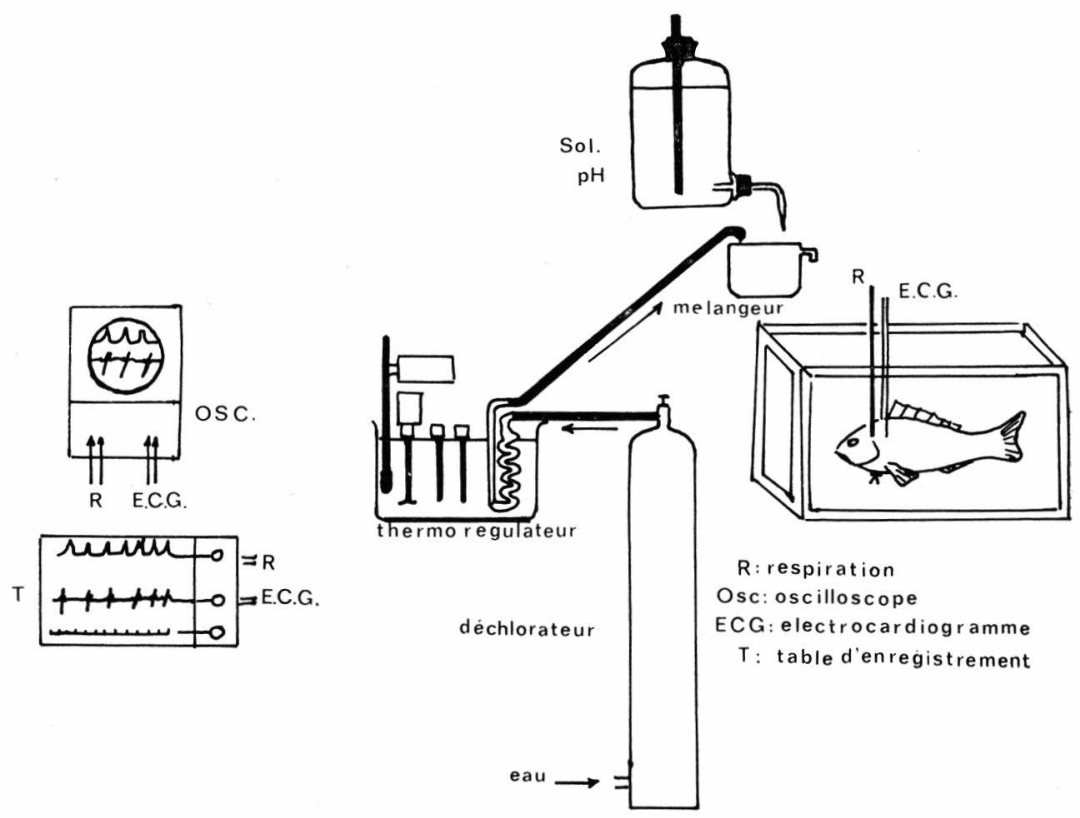

FIG. 1. - Schéma d'installation.

Les variations $\mathrm{du} \mathrm{pH}$ du milieu sont obtenues selon un procédé que nous avons mis au point et qui comprend un flacon de Mariotte contenant une solution acide $(\mathrm{ClH})$ ou alcaline $(\mathrm{NaOH})$ concentrée à la normalité. Un système de goutte à goutte permet à cette solution de tomber en quantité déterminée dans un bac mélangeur où l'on obtient le $\mathrm{pH}$ désiré avec un débit d'eau constant. L'eau utilisée est l'eau de la ville, déchlorée après filtration et amenée à la température voulue par passage dans un ultra-thermostat (fig. 1). Cette eau a été analysée au spectrophotomètre à absorption atomique et ses caractéristiques cationiques sont les suivantes (moyenne sur 7 échantillons) :

$\begin{array}{lcc}\text { Magnésium } & 3,2 & \mu \mathrm{g} / \mathrm{ml} \\ \text { Calcium } & 49 & \mu \mathrm{g} / \mathrm{ml} \\ \text { Zinc } & 0,5 & \mu \mathrm{g} / \mathrm{ml} \\ \text { Plomb } & 0,03 \mu \mathrm{g} / \mathrm{ml} \\ \text { Fer } & 0,1 \mu \mathrm{g} / \mathrm{ml} \\ \text { Cuivre } & 0,03 \mu \mathrm{g} / \mathrm{ml} \\ \text { Résistivité } & 3300 \mu \mathrm{ohms} / \mathrm{cm}\end{array}$


Nous avons expérimenté sur des sujets normaux et vagotomisés placés en eau courante et dont le $\mathrm{pH}$ était maintenu aux valeurs de $5.0-7.2$ et 8.5 , $\mathrm{pH}$ non létaux pour les sujets. Pour chacune de ces valeurs du $\mathrm{pH}$, nous avons fait varier la température de $5{ }^{\circ} \mathrm{C}$ toutes les 48 heures pour des limites comprises entre $20^{\circ}$ et $35{ }^{\circ} \mathrm{C}$.

Les modifications de l'E.C.G. et de la mécanique respiratoire sont suivies à l'oscilloscope et enregistrées systématiquement.

\section{2. - RESUlTATS ELECTROCARDIOGRAPHIQUES}

\section{1. - Chez la Carpe normale.}

2.1.1. - La Fréquence CARDiaque.
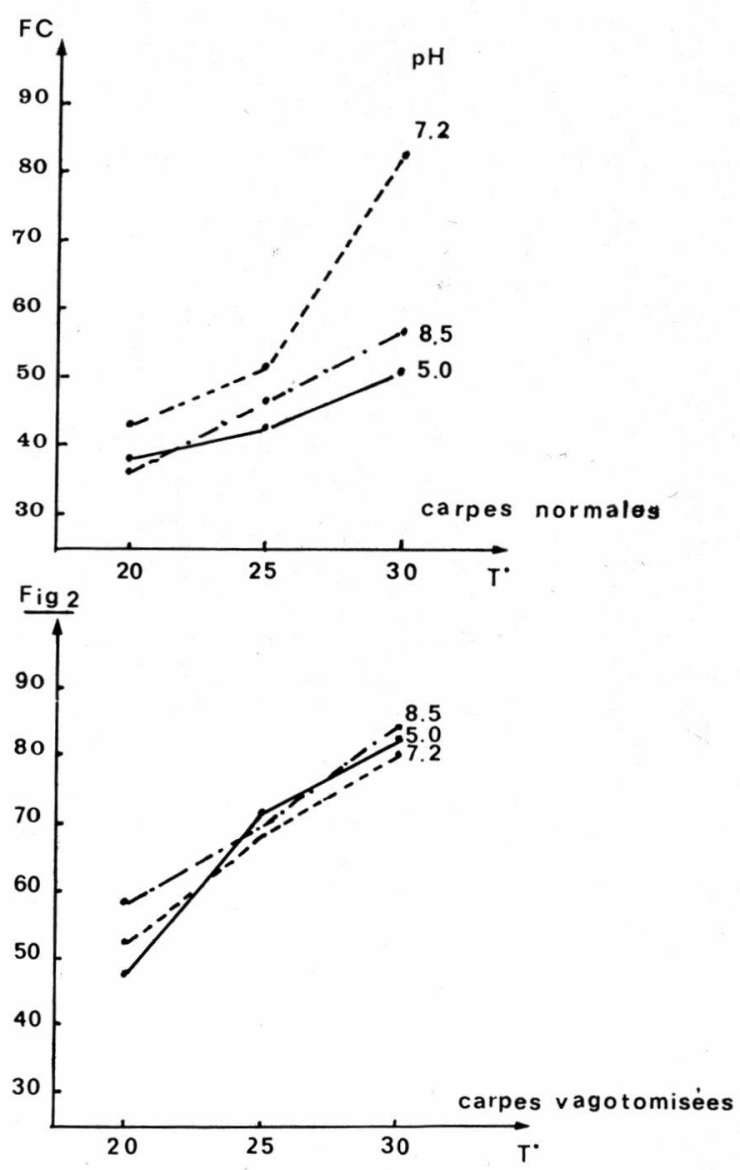

Fig. 2 et 3. - Fréquence cardiaque en fonction de la température à trois niveaux de $\mathrm{pH}$ chez la Carpe. 
Dans un milieu à pH 7.2 , les Carpes adaptées à $20^{\circ} \mathrm{C}$ réagissent par une tachycardie importante à la hausse de la température (Labat 1966). La figure 2 et le tableau I confirment ces résultats (de 42,1 à 81,1 bat $^{s} / \mathrm{mn}$ pour des températures variant entre $20^{\circ}$ et $30{ }^{\circ} \mathrm{C}$ ).

Dans un milieu acide à $\mathrm{pH} 5.0$, la fréquence cardiaque des Poissons ne subit qu'une légère augmentation entre $20^{\circ}$ et $30^{\mathrm{s}} \mathrm{C}$ (de 37,3 à $51 \mathrm{bat}^{\mathrm{s}} / \mathrm{mn}$ ). Au-dela de $30^{\circ} \mathrm{C}$ on observe une accélération particulièrement nette, mais cette tachycardie est cependant moins importante qu'à pH 7.2 (tableau I).

Dans un milieu alcalin à $\mathrm{pH}$ 8.5, les Carpes réagissent aussi à l'augmentation de la température par une tachycardie plus faible qu'à $\mathrm{pH} 7.2\left(57,2 \mathrm{bat}^{\mathrm{s}} / \mathrm{mn}\right.$ à $\left.30^{\circ} \mathrm{C}\right)$, mais plus élevée qu'à $\mathrm{pH} 5$. Cependant il est intéressant de noter qu’à pH 8.5, la température létale des Carpes baisse considérablement par rapport aux résultats observés dans les autres milieux, à pH acide ou proche de la neutralité.

En effet, dans le premier cas, aucun sujet n'a survécu à une température supérieure à $30^{\circ} \mathrm{C}$.

Tableau I. - Comparaison des fréquences cardiaques moyennes aux trois niveaux de pH chez les Carpes et les Tanches.

\begin{tabular}{|c|c|c|c|c|c|}
\hline $\mathbf{T}^{\circ} \mathrm{C}$ & $\mathrm{pH}: 7.2$ & \multicolumn{2}{|c|}{$\begin{array}{c}\mathrm{pH}: 5.0 \\
(\% \text { variation })\end{array}$} & \multicolumn{2}{|c|}{$\begin{array}{c}\mathrm{pH}: 8.5 \\
(\% \text { variation })\end{array}$} \\
\hline \multicolumn{6}{|c|}{ - Carpes normales } \\
\hline $20^{\circ}$ & 42,1 & 37,3 & $(-11,4)$ & 35,9 & $(-14,8)$ \\
\hline $25^{\circ}$ & $\mathbf{5 1 , 9}$ & 43,0 & $(-17,2)$ & 46,7 & $(-10,1)$ \\
\hline $30^{\circ}$ & 81,1 & 51,0 & $(-37,2)$ & 57,2 & $(-29,5)$ \\
\hline $35^{\circ}$ & 96,2 & 75,4 & $(-21,7)$ & & - \\
\hline \multicolumn{6}{|c|}{ - Carpes vagotomisées } \\
\hline $20^{\circ}$ & $\mathbf{5 1 , 9}$ & 46,9 & $(-9,7)$ & 58,0 & $(+11,7)$ \\
\hline $25^{\circ}$ & 67,9 & 71,3 & $(+10,5)$ & 69,0 & $(+1,6)$ \\
\hline $28^{\circ}$ & 80,2 & 82,0 & $(+10,2)$ & 83,3 & $(+3,8)$ \\
\hline \multicolumn{6}{|c|}{ - Tanches normales } \\
\hline $20^{\circ}$ & 47,6 & 37,9 & $(-21)$ & 57,2 & $(+20)$ \\
\hline $25^{\circ}$ & $\mathbf{5 9 , 9}$ & $\mathbf{5 4 , 4}$ & $(-10)$ & 89,7 & $(+49)$ \\
\hline $28^{\circ}$ & 81,4 & 74,2 & $(-9)$ & 109,7 & $(+34)$ \\
\hline \multicolumn{6}{|c|}{ - Tanches vagotomisées } \\
\hline $20^{\circ}$ & 67,0 & 64,8 & $(-4)$ & 80,1 & $(+20)$ \\
\hline $25^{\circ}$ & 110,0 & 93,0 & $(-16)$ & 113,1 & $(+3)$ \\
\hline $28^{\circ}$ & 130,0 & 104,0 & $(-20)$ & 133,5 & $(+2)$ \\
\hline
\end{tabular}




\subsection{2. - LES DÉFLEXIONS ÉLECTROCARDIOGRAPHIQUES.}

Nous avons observé que l'onde $\mathbf{T}$ prend une grande amplitude quand les Carpes se trouvent en milieux acides (pH 5.0). Cette amplitude varie avec la hausse de la température. L'amplitude de l'onde $\mathrm{T}$ augmente jusqu'à $30^{\circ} \mathrm{C}$ puis diminue ensuite entre $30^{\circ}$ et $35^{\circ} \mathrm{C}$. Dans les milieux alcalins $(\mathrm{pH}: 8.5)$, les variations de l'amplitude de l'onde $T$ sont assez irrégulières quand la température augmente.

\subsection{Chez la Carpe vagotomisée.}

\subsection{1. - La FRÉquence CARdiaque.}

Les phénomènes observés chez les Poissons normaux en milieux acides, proches de la neutralité ou alcalins, nous ont incité à penser au rôle possible de la vagotonicité quand l'animal est soumis simultanément aux variations du pH et de la température du milieu ambiant. Nous avons donc expérimenté sur des Carpes ayant subi une vagotomie bilatérale. Un lot de 12 sujets a été utilisé pour chaque série d'expériences, dans les mêmes conditions que les animaux témoins.

Dans un milieu à $\mathrm{pH} 7.2$ le comportement des Poissons vagotomisés peut être résumé par trois faits caractéristiques (fig. 3 et tableau I).

- une fréquence cardiaque plus élevée que chez les Carpes normales aux trois niveaux de température,

- une tachycardie légèrement plus importante, mais surtout plus progressive avec la hausse de la température,

- une température létale plus basse que chez les Carpes normales : $28^{\circ} \mathrm{C}$ environ au lieu de $35^{\circ} \mathrm{C}$.

Chez les sujets vagotomisés, soumis à des milieux acides (5.0) et alcalins (8.5), on observe avec la hausse de la température, une tachycardie bien plus importante que chez les Poissons témoins (fig. 3) et des résultats plus homogènes dans l'ensemble.

\subsection{2. — LES DÉFLEXIONS ÉLECTROCARDIOGRAPHIQUES.}

Chez les Carpes vagotomisées dans un milieu à pH 7.2, le seul phénomène appréciable observé est la diminution de l'amplitude générale de l'E.C.G. avec la hausse de la température.

\section{3. - Chez la Tanche normale.}

\subsection{1. - La FRÉquence CARdiaqu.}

Dans un milieu à $\mathrm{pH} 7.2$, les Tanches témoins adaptées à $20^{\circ} \mathrm{C}$ réagissent par une tachycardie très importante à la hausse de la température (de $47,6 \mathrm{bat}^{\mathrm{s}} / \mathrm{mn}$ à $20^{\circ} \mathrm{C}$ et de $81,4 \mathrm{bat}^{\mathrm{s}} / \mathrm{mn}$ à $28{ }^{\circ} \mathrm{C}$ ) (fig. 4, tableau I). 

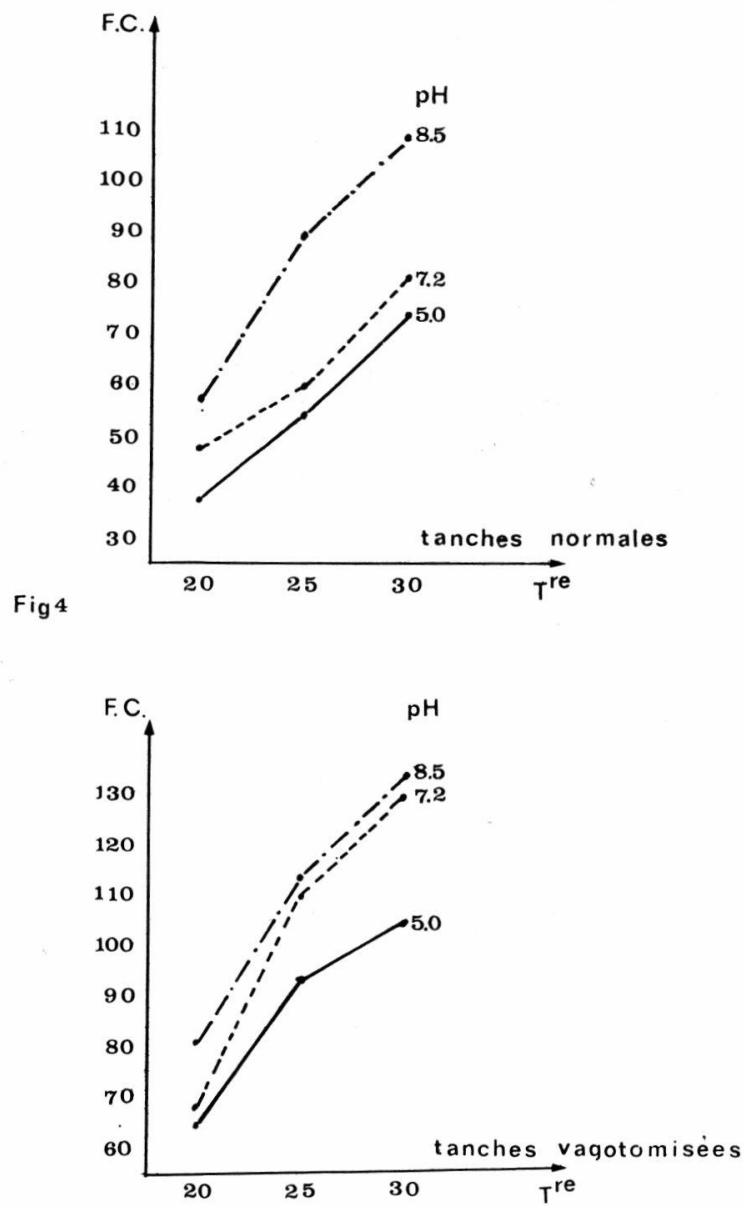

Fig. 4 et 5. - Fréquence cardiaque en fonction de la température à trois niveaux de $\mathrm{pH}$ chez la Tanche.

En milieu acide ( $\mathrm{pH}$ 5.0) la fréquence cardiaque ne subit qu'une augmentation modérée (fig. 4, tableau I), par rapport à celle que l'on constate à $\mathrm{pH} 7.2$.

En milieu alcalin ( $\mathrm{pH}:$ 8.5) nous observons aussi une tachycardie. Mais son intensité est supérieure à celle qui caractérise les milieux proches de la neutralité ( $\mathrm{pH}: 7.2$ ).

\subsection{2. - LES DÉFLEXIONS ÉLECTROCARDIOGRAPHIQUES.}

D'une façon générale, il semble qu'il y ait une légère diminution de l'amplitude de l'E.C.G. avec l'augmentation de la température et cela quel que soit le $\mathrm{pH}$ du milieu ambiant. 


\section{4. - Chez la Tanche vagotomisée.}

\subsection{1. - LA FRÉQUENCE CARdiaque.}

Une fréquence cardiaque plus élevée que chez les sujets normaux, semble caractéristique chez les Poissons vagotomisés (Labat, 1966). De ce fait la tachycardie provoquée par la hausse de la température arrive à être considérable $\left(130 \mathrm{bat}^{\mathrm{s}} / \mathrm{mn}\right)$ (fig. 5 et tableau I).

Il faut cependant noter que le milieu acide ( $\mathrm{pH}$ : 5.0) semble provoquer ici aussi une influence modératrice sur la physiologie cardiaque des Poissons (104 bat $/ \mathrm{mn})$.

On observe par contre des fréquences très élevées $\left(133,5 \mathrm{bat}^{\mathrm{s}} / \mathrm{mn}\right)$ en milieu alcalin ( $\mathrm{pH}: 8)$.

\subsection{2. - LES DÉFLEXIONS ÉLECTROCARDIOGRAPHIQUES.}

Dans toutes les expériences et aux trois niveaux de $\mathrm{pH}$, nous avons remarqué une légère diminution de l'amplitude du complexe électrocardiographique avec l'augmentation de la température.

\section{3. - RESULTATS CONCERNANT LES MOUVEMENTS OPERCULAIRES}

L'ensemble de nos expériences montre clairement qu'à pH 7.2 et à $\mathrm{pH} \mathrm{8.5,} \mathrm{les} \mathrm{résultats} \mathrm{sont} \mathrm{homogènes,} \mathrm{c'est-à-dire} \mathrm{que} \mathrm{les}$ fréquences respiratoires ont des valeurs proches de celles des fréquences cardiaques. La lecture du tableau II et surtout les courbes de la figure 6 illustrent bien ce phénomène. Au contraire, dans les milieux acides ( $\mathrm{pH}: 5.0)$, il existe une dissociation nette entre les fréquences de ces deux mécanismes physiologiques. En effet, alors que le rythme cardiaque est relativement ralenti comme nous en avons fait l'observation plus haut, la fréquence des mouvements operculaires s'élève normalement avec la température (tableau II; fig. 6).

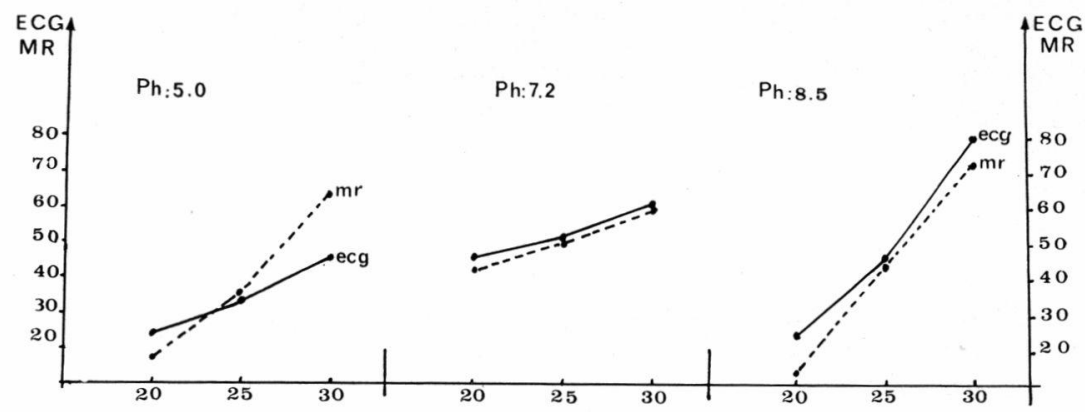

Fig. 6. - Fréquences cardiaque et respiratoire à trois niveaux de $\mathrm{pH}$ chez les Carpes. 


\section{4. - INFLUENCE DU $\mathrm{pH}$ DU MILIEU EXTÉRIEUR SUR LE pH SANGUIN}

Nous avons opéré sur 15 Carpes normales et 15 Carpes vagotomisées d'un poids moyen de 400 à $500 \mathrm{~g}$, ainsi que sur 15 Tanches normales et 15 Tanches vagotomisées d'un poids moyen de 150 à 200 g. Les Poissons sont mis dans les mêmes conditions expérimentales que les précédents.

Les prélèvements sanguins sont effectués par ponctions intracardiaques à l'aide de seringues héparinées. Chaque prélèvement est d'environ 1/10 de $\mathrm{ml}$ afin de pouvoir faire deux ou trois mesures successives. Ces dernières sont pratiquées par analyseur de $\mathrm{pH}$ et des gaz du sang (modèle 213/227 I.L.). Les mesures sanguines de la $\mathrm{PO}^{2}$ et de la $\mathrm{PCO}^{2}$ seront effectuées ultérieurement.

\section{- Chez les Carpes normales.}

Chez tous les sujets, nous avons observé une influence plus ou moins considérable des variations de la température et du $\mathrm{pH} d u$ milieu extérieur sur le $\mathrm{pH}$ sanguin.

Nous remanquerons que les résultats sont plus homogènes pour les milieux acides que pour les milieux proches de la neutralité ou alcalins (tableau III).

- chez les Carpes vagotomisées.

Dans ces expériences nous ferons aussi la même remarque que précédemment, toutefois le facteur individuel semble plus important (tableau III). Nous serons donc obligés d'utiliser les méthodes statistiques pour pouvoir discuter ces résultats.

- chez les Tanches normales.

Chez les Tanches, c'est aussi en milieu acide ( $\mathrm{pH}: 5.0)$ que nous observons les résultats les plus homogènes. Dans les milieux proches de la neutralité ou alcalins, les influences individuelles sont vraiment importantes (tableau III).

- chez les Tanches vagotomisées.

Les résultats ici sont assez décevants car il est difficile de les interpréter. Nous pensons que ces expériences doivent être reprises avec un plus grand nombre d'individus (tableau III).

\section{5. - ANALYSE}

\section{DES RESULTATS ÉLECTROCARDIOGRAPHIQUES}

S'il existe un effet d'interaction dans l'analyse de variance, c'est qu'au moins une des deux variables $\left(t^{\circ}\right.$ ou $\left.\mathrm{pH}\right)$ a un effet certain, mais cela ne signifie pas nécessairement que ces deux variables aient un effet simultané. Dans le cas où $F$ de l'interaction est trop grand pour nous permettre d'accepter l'hypothèse du hasard, le 
carré moyen de l'interaction permettra de tester séparément les variations de la température et du $\mathrm{pH}$, et d'obtenir ainsi l'effet spécifique de chaque variable.

- chez la Carpe normale (tableau IV).

Les résultats obtenus pour chacune des composantes de la variance totale sont significatifs ${ }^{1}$ et ceux qui correspondent à la température et au $\mathrm{pH}$ sont très significatifs :

$$
F_{e x}(27,27)>>>F_{0 \cdot 1}(4,61) \text { et } F_{e x}(10,62)>>F_{0.1}(4,61) .
$$

Cependant, il faut observer que la variabilité individuelle est aussi significative.

En tenant compte de l'interaction, on peut en déduire que l'effet spécifique de la température se maintient avec un intervalle de confiance de $99 \%$, tandis que l'effet du $\mathrm{pH}$ a une signification au seuil de $5 \%$ seulement.

Par conséquent, nous pouvons dire que dans nos expériences sur la Carpe, la température est le facteur prépondérant.

- chez la Carpe vagotomisée (tableau IV).

Tous les $\mathrm{F}$ correspondant aux blocs et à la température sont plus grands que l'unité. Seul le facteur température est très significatif. $F_{\text {ex }}(43,47)>>F_{0.1}(4,98)$.

Par conséquent, nous pouvons penser que les variations observées sur la fréquence cardiaque pendant les expériences sont surtout dues à la température. D'autre part, la variabilité individuelle des sujets en expériences est plus significatives que l'effet du $\mathrm{pH}$.

- chez la Tanche normale et vagotomisée (tableau IV).

Les exemplaires utilisés dans les expériences ont donné des résultats d'ensemble bien plus homogènes que ceux des Carpes. En effet, les tests pour cette composante de l'analyse de la variance (blocs) ne sont pas significatifs, tant pour les Tanches normales que pour les vagotomisées.

Nous avons observé aussi que les deux facteurs température et $\mathrm{pH}$ ont chacun des effets très significatifs sur la fréquence cardiaque chez les Tanches normales :

$$
F_{e x}(74,56)>>>F_{0.1}(4,79) \text { et } F_{\text {ex }}(43,71)>>>F_{0.1}(4,79)
$$

et pour les Tanches vagotomisées :

$$
F_{e x}(46,0)>>>F_{0.1}(4,98) \text { et } F_{e x}(8,0)>>F_{0.1}(4,98) \text {. }
$$

1. Remarque : Dans les tableaux, les résultats très significatifs $\left({ }^{\star *}\right)$ correspondent au seuil de $1 \%$; les résultats significatifs (*) correspondent au seui] de $5 \%$. 


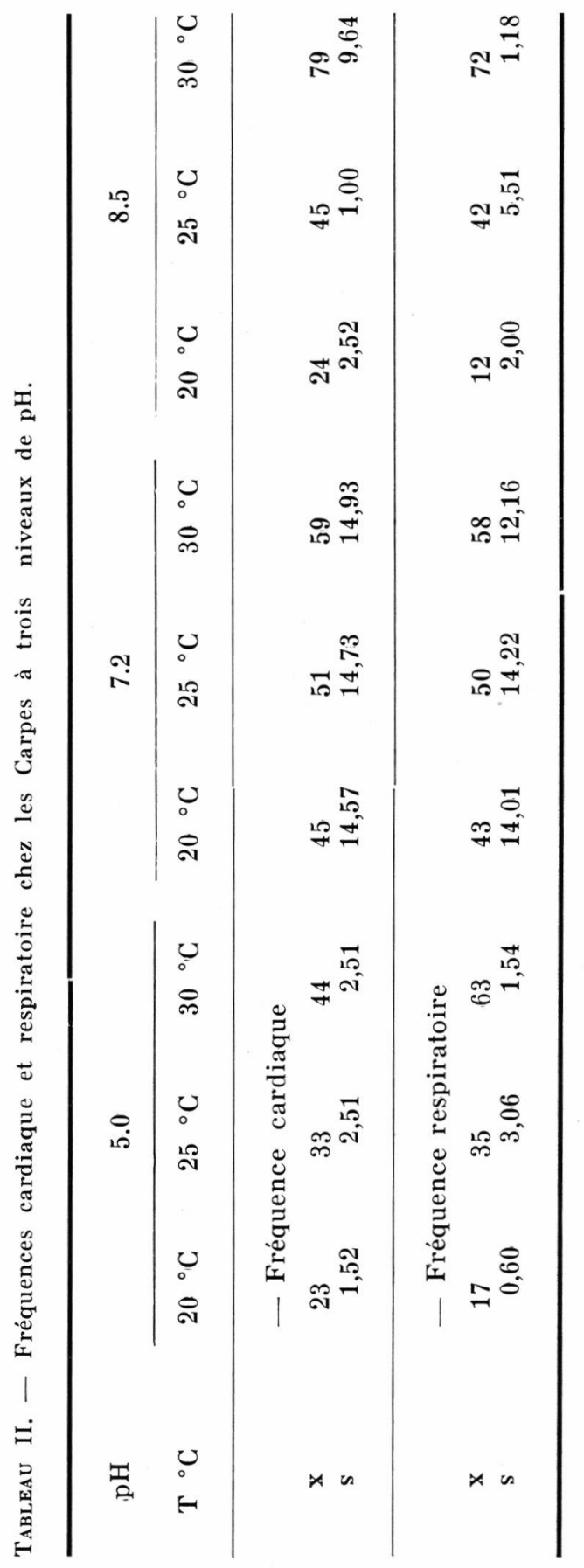


G. DAZAROLA, R. LABAT ET J. KUGLER

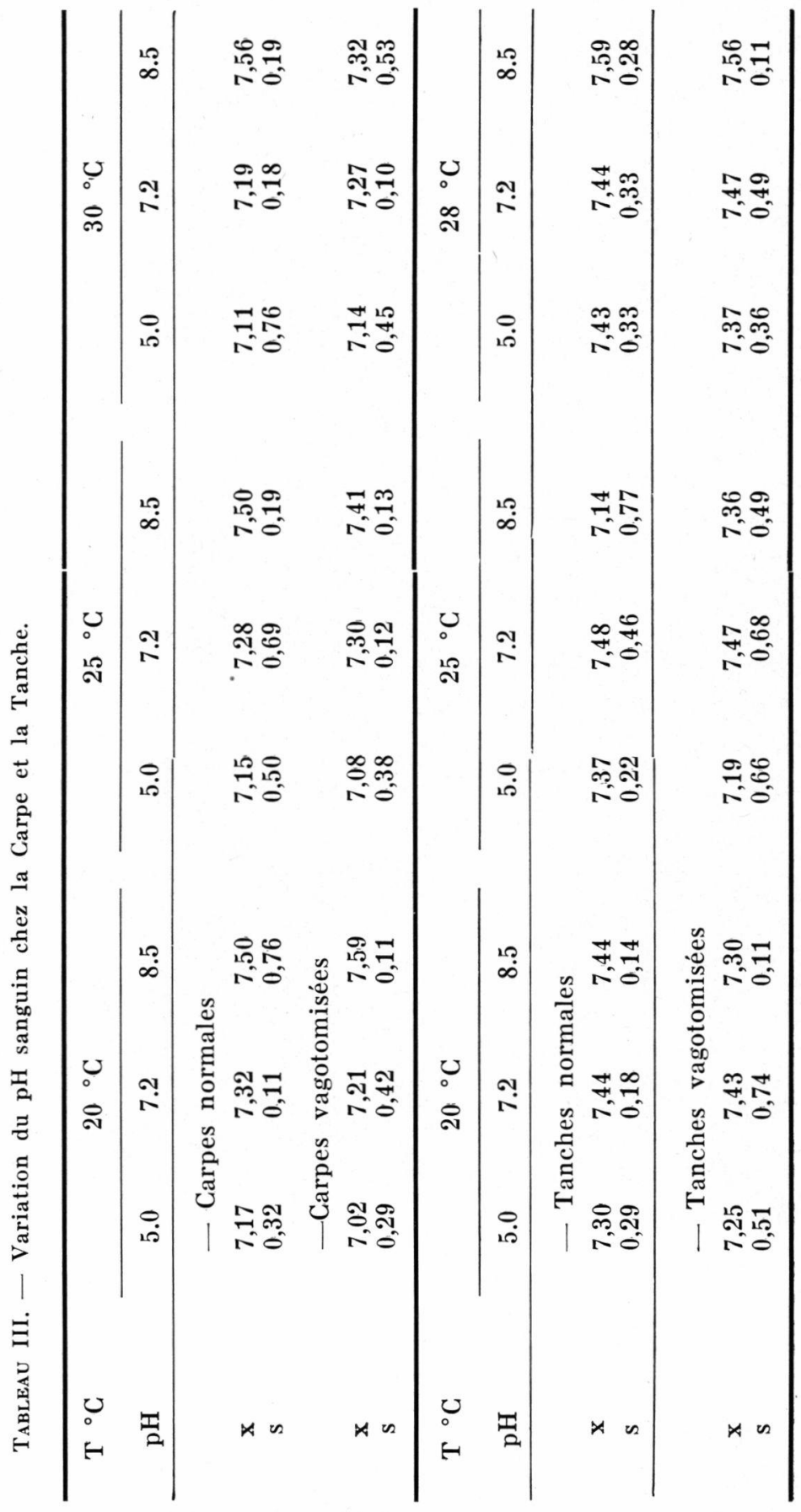


Tableau IV. - Valeurs de « $F »$ obtenues dans les analyses de variance.

\begin{tabular}{lcccc}
\hline & $\begin{array}{c}\text { Carpes } \\
\text { normales }\end{array}$ & $\begin{array}{c}\text { Carpes } \\
\text { vagotomisées }\end{array}$ & $\begin{array}{c}\text { Tanches } \\
\text { normales }\end{array}$ & $\begin{array}{c}\text { Tanches } \\
\text { vagotomisées }\end{array}$ \\
\hline Blocs & 3,30 & $2,18^{*}$ & $1,42 \mathrm{NS}$ & $0,48 \mathrm{NS}$ \\
Traitement & $27,27^{* *}$ & $43,37^{* *}$ & 74,56 & $46,0^{* *}$ \\
Traitement pH & $10,62^{* *}$ & $0,75 \mathrm{NS}$ & 43,71 & $8,0^{* *}$ \\
Intéraction & $3,18^{*}$ & $0,81 \mathrm{NS}$ & $2,14 \mathrm{NS}$ & $0,9 \mathrm{NS}$ \\
\hline
\end{tabular}

\section{6. - DISCUSSION}

Aux températures expérimentales de $20-25-30^{\circ} \mathrm{C}$ chez les Carpes, et de $20-25-28{ }^{\circ} \mathrm{C}$ chez les Tanches, les effets électrocardiographiques et respiratoires sont différents selon que les sujets sont placés en milieu acide ( $\mathrm{pH}: 5.0)$, en milieu proche de la neutralité ( $\mathrm{pH}: 7.2$ ), ou alcalin ( $\mathrm{pH}: 8.5$ ). L'étude des variations des deux facteurs du milieu, température et $\mathrm{pH}$, montre qu'il existe une grande complexité dans les réactions physiologiques.

Cependant l'analyse factorielle nous permet de penser que les variations du facteur température sont plus importantes que celles dévolues au facteur $\mathrm{pH}$, ou que celles dues à l'interaction des deux facteurs.

En effet, au-dessous de $25^{\circ} \mathrm{C}$, chez la Carpe, en milieu acide, l'élévation de la température du milieu produit peu de perturbations électrocardiographiques et respiratoires. En milieu alcalin, ces perturbations sont plus appréciables. En outre, la double vagotomie lève les effets inhibiteurs dus au milieu acide ou alcalin visà-vis des variations thermiques. Ainsi, le rôle du parasympathique dans les échanges ioniques entre le Poisson et son milieu est mis en relief, en particulier jusqu'à la température de $25^{\circ} \mathrm{C}$.

$\mathrm{Au}$-dessus de $25^{\circ} \mathrm{C}$, chez la Carpe, en milieu alcalin, les modifications électrocardiographiques et operculaires suivent les variade la température ambiante. En milieu acide, on observe un disfonctionnement entre centres cardiaques et respiratoires. La polypnée s'accentue avec la hausse de la température, tandis que le rythme cardiaque reste relativement lent. Cette polypnée semble conforme aux travaux de Randall et coll. (1966-1970) qui ont observé une consommation accrue d'oxygène chez Salvelinus fontinalis placé en milieu acide. D'autre part, Tan et coll. (1972) ont démontré que l'affinité de l'hémoglobine de Carpe pour l'oxygène est 160 fois plus importante en $\mathrm{pH}$ alcalin q'en $\mathrm{pH}$ acide.

Ces phénomènes étant liés au pH du milieu intérieur, nous avons pu constater que, chez les Carpes normales, le $\mathrm{pH}$ sanguin dépend principalement des variations $\mathrm{du} \mathrm{pH}$ du milieu ambiant. Chez les 
Carpes vagotomisées, le $\mathrm{pH}$ sanguin est soumis plus directement aux variations thermiques. Ces résultats semblent être en accord avec les travaux de Péquignot (1972) qui constate chez les Poissons vagotomisés une augmentation du volume plasmatique et donc du Na total sanguin. Ceci pourrait être dû à une augmentation de la réserve alcaline du milieu intérieur permettant une meilleure régulation $\mathrm{du} \mathrm{pH}$.

Notons que si les variations du $\mathrm{pH}$ sanguin chez les Mammifères sont très limitées $(7.39-7.43)$, ce qui implique une homéostasie très poussée, il n'en est pas ainsi chez la Carpe ou la Tanche où les oscillations $\mathrm{du} \mathrm{pH}$ sanguin varient entre 6.90 et 7.90 .

Il semble done intéressant de poursuivre des recherches, pour élucider les mécanismes de l'équilibre acido-basique chez les Poissons qui montrent une grande souplesse par rapport aux vertébrés supérieurs. D'après Dejours (1966), l'énorme différence entre la solubilité de l'oxygène et du gaz carbonique, qui caractérise le milieu aquatique, doit entraîner une relation entre la concentration du $\mathrm{CO}_{2}$ et la $\mathrm{pCO}_{2}$. Or chez les Poissons, seules des concentrations élevées en $\mathrm{CO}_{2}$ semblent agir efficacement, tandis que de faibles variations du taux d'oxygène dissous modifient sensiblement le tracé respiratoire (Peyraud, 1965).

Enfin il nous paraît important de noter la plus grande homogénéité des résultats fournis par les Tanches, tandis que le facteur individuel semble dominer chez les Carpes.

\section{TRAVAOX CITÉS}

Dazarola (G.), Labat (R.) et Serfaty (A.). 1971. - L'enregistrement des mouvements operculaires chez un Téléostéen nageant librement dans son milieu naturel. Bull. Soc. Hist. Natur. Toulouse, $107,3-4,437-441$.

Desours (P.). 1966. - Respiratory gas exchange of aquatic animals during confinement. J. Physiol. (London), 186, 126-127.

Dussart (B.). 1966. - Limnologie. L'étude des eaux continentales. Gauthier-Villars, 677 pages.

I.abat (R.) et Serfaty (A.). 1963. - Electrocardiographie et vagotomie chez la Carpe commune (Cyprinus carpio L.). Bull. Soc. Hist. Nat. Toulouse, 98, 1-2, 192-196.

Labat (R.). 1966. - Electrocardiographie chez les Poissons Téléostéens. Influence de quelques facteurs écologiques. Annls. Limnol., 2, 1-175.

Iabat (R.), Kugler-Laffont (J.) et Cadastraing (A.). 1969. - Le pH du milieu ambiant et ses effets sur l'électrocardiogramme de quelques Téléostéens dulçaquicoles — première partie. Bull. Soc. Hist. Nat. Toulouse, 105, 1-2, 167-180.

Labat (R.), Kugler-Laffont (J.), Cadastraing (A.) et Bonnet (L.). 1969. - Le pH du milieu ambiant et ses effets sur l'électrocardiogramme de quelques Téléostéens dulçaquicoles - deuxième partie. Bull. Soc. Hist. Nat. Toulouse, 105, 3-4, 455-463. 
Pequignot (J.). 1972. - Réactions comparées de deux Téléostéens, Tanche et Anguille, lors d'un changement de salinité : influence du parasympathique. Thèse de l'Université Paul Sabatier 165 pages.

Peyraud (C.). 1965. - Recherches sur la régulation des mouvements respiratoires chez quelques Téléostéens : analyse d'un réflexe opto-respiratoire. Thèse de l'Université Paul Sabatier, Toulouse, 260 pages.

Randall (D. J.), Holeton (G. F.) et Don Stevens (E.). 1966. - The exchange of oxygen and carbon dioxide across the gills of rainbow trout. J. Exp. Biol., 6, 339-348.

Randall (K. P.) et Dunson (W. A.). 1970. - Effets of low environmental $\mathrm{pH}$ on blood $\mathrm{pH}$ and sodium balance of brook trout. J. Exp. Zool., 174, 1, 65-72.

'Tan (A. L.), Young (A. D.) et Noble (R. W.). 1972. - The pH dependance of the affinity kinetics, and cooperativity of ligan brinding to carp hemoglobin, Cyprinus carpio L. J. Biol. Chem., 247, 2493-98. 